\title{
ON THE RAMIFICATION THEORY OF REGULAR SCHEMES
}

\author{
ZHAOHUA LUO
}

(Communicated by Eric Friedlander)

\begin{abstract}
We prove a fundamental theorem on the ramification of morphisms of regular schemes, extending Dedekind's theorem of different.
\end{abstract}

Suppose $f: X \rightarrow Y$ is a dominant morphism of smooth varieties unramified at the generic point (e.g., a birational morphism). Classically the ramification divisor $E_{f}$ of $f$ is defined via canonical sheaves, i.e., $E_{f}=\operatorname{div}\left(f^{*}\left(\omega_{X} \otimes \omega_{Y}^{-1}\right)\right)$ (cf. [D]). If $X, Y$ are regular schemes and $f$ is essentially of finite type as above, then $E_{f}$ can be defined to be the effective divisor determined by the Kähler different (sheaf) of the morphism $f$ (cf. [L2]).

For any $x \in X$ and $y=f(x) \in Y$ we shall introduce three important invariants $r(x y), e(x y)$, and $w(x y)$, where $r(x y)$ is the multiplicity of $E_{f}$ at $x$ and $e(x y)$ is the supremum of the multiplicities at $x$ of the products of local coordinates of $Y$ at $y$. The main purpose of this paper is to prove the following theorem, which is a generalization of the main theorem of ramification theory of algebraic number theory (due to Dedekind).

Theorem 1 (Geometric form). Let $f: X \rightarrow Y$ be a morphism of regular schemes as above. Then for any $x \in X$ and $y=f(x)$ we have

$$
\begin{aligned}
r(x y) & \geq e(x y)-\operatorname{codim} x \geq w(x y)+\operatorname{codim} y-\operatorname{codim} x \\
& \geq \operatorname{codim} y-\operatorname{codim} x \geq 0 .
\end{aligned}
$$

(i) Suppose $f$ is a finite morphism and $\operatorname{codim} x=\operatorname{codim} y=1$. Then $r(x y)=e(x y)-1$ if and only if the residue field $k(x)$ of $x$ is a separable extension of the residue field $k(y)$ of $y$ and $e(x y)$ is not a multiple of the characteristic of $k(y)$.

(ii) Suppose $f$ is a birational morphism. Then $r(x y)=\operatorname{codim} y-\operatorname{codim} x$ if and only if $x$ and $y$ determine the same discrete valuation of the rational function field; in this case we have $m_{y} \mathscr{O}_{x}=m_{x}$ (thus $y$ is the generic point of a component of $\left.f^{-1}(x)\right)$.

The expression $(*)$ is one of the most important formulas for the ramification theory of regular schemes (see [L, L1, L3] for applications); a preliminary

Received by the editors March 12, 1992.

1991 Mathematics Subject Classification. Primary 14E22, 13 H05.

Supported partially by the Tian Yuan Foundation. 
version of $(*)$ (i.e., $r(A B) \geq e(A B)-1$ for the case $\operatorname{codim} x=1$ ) was announced in [L1] and proved in [L2].

Since the ramification theory of regular schemes is essentially a local problem, we shall carry out our study mainly in the category of commutative algebras. Theorem 1 is proved in its algebraic form at the end of the paper. We first introduce some notation and terminology.

In this paper by a pair $(A, B)$ of regular local rings we always mean a dominating pair $A \supseteq B$ of regular local rings such that the quotient field $Q(A)$ of $A$ is a finite separable extension of the quotient field $Q(B)$ of $B$ and $A$ is a quotient ring of a finitely generated $B$-algebra (i.e., $A$ is a $B$-algebra essentially of finite type). Denote by $M$ and $N$ the maximal ideals of $A$ and $B$ respectively.

Denote by $D(A / B)$ the differential module of $A$ over $B . D(A / B)$ is a finitely generated torsion $A$-module and the 0-Fitting ideal $d(A / B)$ of $D(A / B)$ is a nonzero principal ideal of $A$.

If $P$ is any subset of a local ring $A$, we define

$$
\operatorname{ord}_{A}(P)=\sup \left\{t \in \mathbb{Z} \mid P \subset M^{t}\right\} \in \mathbb{Z} \cup \infty .
$$

If $A$ is a regular local ring then ord $_{A}$ determines a discrete valuation $v_{A}$ of the quotient field $Q(A)$ of $A$; denote by $Z(A)$ the discrete valuation ring of $v_{A}$. If $E=\left\{b_{1}, \ldots, b_{s}\right\}$ is a finite subset of $A$, for simplicity we shall write $I(E)$ for the product $b_{1} \cdot b_{2} \cdots b_{s}$ of these elements.

Definition 1. Suppose $(A, B)$ is a pair of regular local rings with $\operatorname{dim} A=m$ and $\operatorname{dim} B=n$ (here $\operatorname{dim} A=$ krull $\operatorname{dim} A$ ). We define

$$
\begin{aligned}
& r(A B)=v_{A}(d(A / B)) ; \\
& e(A B)=\sup \left\{v_{A}(I(E)) \mid E \text { is a minimal basis of } N\right\} \\
& w(A B)=\sup \left\{t\left(v_{A}(Q)-1\right) \mid Q \text { is a prime ideal of } B \text { of height } t\right. \\
& s(A B)=(n-m) v_{A}(N) .
\end{aligned}
$$

$r(A B)$ and $e(A B)$ are called the ramification index and the reduced ramification index of $A$ over $B$ respectively. (Clearly we have $e(A B)-m \geq w(A B)+n-m \geq$ $s(A B) \geq n-m \geq 0$. We shall prove that $e(A B)<\infty$.)

Let $\mathscr{C}(A / B)$ be the set of prime ideals of $A$ of height 1 such that $P$ is ramified over $B$ (i.e., $A_{P}$ is ramified over $\left.B_{P \cap B}\right) ; \mathscr{C}(A / B)$ is a finite set.

The reader is referred to [K1, K2, L2] for the general theory of Kähler different.

In the following we assume that $(A, B),(B, C)$, and $(C, D)$ are pairs of regular local rings (then $(A, C),(A, D)$, and $(B, D)$ are also pairs of regular local rings).

Lemma 1. $r(A B)=0$ if and only if $A$ is unramified over $B$. If $Q(A)=Q(B)$, then $r(A B)=0$ if and only if $A=B$.

Droof. $A$ is unramified if and only if $d(A / B)=(1)$, i.e., $r(A B)=0$ (see [K1]). If $Q(A)=Q(B)$ then $A$ is unramified over $B$ if and only if $A=B$; thus $r(A B)=0$ if and only if $A=B$ in this case. 
Lemma 2. $r(A B)=\sum_{P \in \mathscr{C}(A / B)} r\left(A_{P} B_{P \cap B}\right)\left(v_{A}(P)\right)$. Thus $r(A B) \geq|\mathscr{C}(A / B)|$.

Proof. Since $d(A B)$ is a principal nonzero ideal of $A, d(A B)=\prod a_{i}^{t_{i}}$ with $a_{i} \in A$ irreducible. Then $\mathscr{C}(A / B)$ consists of all the prime ideals $P_{i}=\left(a_{i}\right) A$. We have $v_{A}\left(P_{i}\right)=v_{A}\left(a_{i}\right)$ and $r\left(A_{P_{i}} / B_{P_{i} \cap B}\right)=t_{i}$. Thus $r(A B)=v_{A}(d(A / B))=$ $\sum\left(v_{A}\left(a_{i}\right)\right) t_{i}=\sum_{P \in \mathscr{C}(A / B)} r\left(A_{P} B_{P \cap B}\right)\left(v_{A}(P)\right)$.

Lemma 3. Write $r(A B C)=v_{A}(d(B / C))$. Then we have $r(A C)=r(A B)+$ $r(A B C)$. Also $r(A B C)=\sum_{P \in \mathscr{C}(B / C)} r\left(B_{P} C_{P \cap C}\right)\left(v_{A}(P)\right)$.

Proof. We have $d(A / C)=d(A / B)(d(B / C) A)$ [L2]; thus $r(A C)=r(A B)+$ $r(A B C)$. The proof of the second assertion is similar to that of Lemma 2.

Lemma 4. $r(A B C) \geq r(B C)$ and $r(Z(B) B C)=r(B C)$. Thus $r(A C) \geq$ $r(A B)+r(B C)$.

Proof. Note that $v_{A}(P) \geq v_{B}(P)$ for any subset $P$ of $B$ and $v_{Z(B)}=v_{B}$.

Lemma 5. $r(A B D)=r(A B C)+r(A C D)$ where $r(A B D)$ and $r(A C D)$ have the same meaning as $r(A B C)$.

Proof. We have $r(A B D)=r(A D)-r(A B)=(r(A C)+r(A C D))-r(A B)=$ $(r(A C)-r(A B))+r(A C D)=r(A B C)+r(A C D)$.

Lemma 6. Suppose $A$ is a discrete valuation ring and $B$ is the first quadratic transform of $C$ along $A$ (see [A]). Then $e(A C) \leq e(A B)+s(A C)$ and $r(A B C)=$ $s(A C)$.

Proof. Let $E=\left\{b_{1}, \ldots, b_{t}\right\}$ be a minimal basis of the maximal ideal $Q$ of $C$. Suppose we have arranged the $b_{i}$ so that $v_{A}\left(b_{1}\right)=\cdots=v_{A}\left(b_{t^{\prime}}\right)<v_{A}\left(b_{t^{\prime}+1}\right) \leq$ $\cdots \leq v_{A}\left(b_{t}\right)$. Then $v_{A}(Q)=v_{A}\left(b_{1}\right)$, which implies that $s(A C)=(t-1) v_{A}\left(b_{1}\right)$. The set $E^{\prime}=\left\{b_{1}, b_{t^{\prime}+1} / b_{1}, \ldots, b_{t} / b_{1}\right\}$ is part of a minimal basis of the maximal ideal $N$ of $B$; hence $e(A B) \geq v_{A}\left(I\left(E^{\prime}\right)\right)$. Since $v_{A}\left(b_{i}\right)=v_{A}\left(b_{1}\right)$ for $i \leq t^{\prime}$, we have $v_{A}(I(E))=v_{A}\left(I\left(E^{\prime}\right)\right)+(t-1) v_{A}\left(b_{1}\right) \leq e(A B)+s(A C)$ for any minimal basis $E$ of $Q$. It follows that $e(A C) \leq e(A B)+s(A C)$. As $B$ is a quadratic transform of $C$, we have $d(B / C)=Q^{t-1} B$ (cf. [L2]); hence $r(A B C)=v_{A}(d(B / C))=(t-1) v_{A}(Q)=s(A C)$.

Suppose $Q(A)=Q(B)$ and $A$ is a discrete valuation ring. Consider the quadratic sequence along $A$ starting from $B$ such that each $B_{i}$ is the first quadratic transform of $B_{i-1}$ along $v_{A}$ (cf. [A, p. 336]):

$$
A=B_{t} \supset \cdots \supset B_{2} \supset B_{1} \supset B_{0}=B \text {. }
$$

The existence of such a finite sequence is guaranteed by Lemma 3 , as the length $t$ of any such strictly descending chain between $A$ and $B$ is bounded by $r(A B)$, because we have $r(A B)=\sum_{i=0, \ldots, t-1} r\left(A B_{i+1} B_{i}\right) \geq t$.

Lemma 7. Suppose $Q(A)=Q(B)$ and $A$ is a discrete valuation ring. Then

(i) $r(A B)=\sum_{i=0, \ldots, t-1} s\left(A B_{i}\right) \geq e(A B)-1$.

(ii) The following assertions are equivalent:

(a) $A=Z(B)$;

(b) $r(A B)=s(A B)$;

(c) $r(A B)=n-1$.

Proof. (i) Note that $s\left(A B_{i}\right)=r\left(A B_{i+1} B_{i}\right)$ by Lemma 6; hence $r(A B)=$ $\sum_{i=0, \ldots, t-1} r\left(A B_{i+1} B_{i}\right)=\sum_{i=0, \ldots, t-1} s\left(A B_{i}\right)$. Applying Lemma 6 and using 
induction on $t$ we see that $e(A B)$ is finite. Also by that lemma we obtain $r(A B)=\sum_{i=0, \ldots, t-1} s\left(A B_{i}\right) \geq \sum_{i=0, \ldots, t-1} e\left(A B_{i}\right)-e\left(A B_{i+1}\right)=e(A B)-$ $e\left(A B_{t}\right)=e(A B)-1$.

(ii) Write $c=\sum_{i=1, \ldots, t-1} s\left(A B_{i}\right)$. Then $c=0$ if and only if $A=Z(B)$. But $r(A B)=c+s(A B) \geq c+n-1$. Thus $c=0$ if and only if $r(A B)=s(A B)$. Thus (a) and (b) are equivalent. If $r(A B)=n-1$ then $c=0$; thus $A=Z(B)$. Clearly $A=Z(B)$ implies $r(A B)=n-1$. Thus (a) and (c) are equivalent.

Remark. The formula $r(A B)=\sum_{i=0, \ldots, t-1} s\left(A B_{i}\right)$ gives another definition of $r(A B)$ for any birational pair $(A, B)$ of regular local rings such that $\operatorname{dim} A=1$, without referring to Kähler different. If $A$ and $B$ are regular localities of an algebraic function field $K / k$ then one can use this formula to prove that our definition of $r(A B)$ coincides with the usual one obtained by means of the canonical divisors of $K / k$ (see [L2]).

Lemma 8. $r(A B)=r(Z(A) B)-m+1$.

Proof. We have $r(A B)=r(Z(A) A B)=r(Z(A) B)-r(Z(A) A)=r(Z(A) B)-$ $(m-1)$ by Lemmas 3, 4, and 7(ii).

Theorem 2 (Algebraic form). For any pair $(A, B)$ of regular local rings we have

$$
\begin{aligned}
r(A B) & \geq e(A B)-\operatorname{dim} A \geq w(A B)+\operatorname{dim} B-\operatorname{dim} A \\
& \geq s(A B) \geq \operatorname{dim} B-\operatorname{dim} A \geq 0 .
\end{aligned}
$$

(i) Suppose $A, B$ are discrete valuation rings; then $r(A B)=e(A B)-1$ if and only if $e(A B)$ is not a multiple of $\operatorname{ch} B / N$, and $A / M$ is a separable extension of $B / N$.

(ii) Suppose $Q(A)=Q(B)$; then $r(A B)=0$ if and only if $A=B$, and $r(A B)=\operatorname{dim} B-\operatorname{dim} A$ if and only if $Z(A)=Z(B)$. If $Z(A)=Z(B)$ then $N A=M$.

Proof. (i) If $A, B$ are discrete valuation rings, then $d(A B)$ coincides with the Dedekind different of $A$ over $B$ (see [L2]), and $r(A B)$ is called the differential exponent in [ZS]. Thus (i) is the content of Dedekind's theorem of different.

To prove (\#) we consider $r(A B)=r(Z(A) B)-m+1$ (Lemma 8). If we can prove $r(Z(A) B) \geq e(Z(A) B)-1$, then, since $e(Z(A) B)=e(A B)$, we would obtain $r(A B) \geq e(A B)-m$. Thus we may assume that $A$ is a discrete valuation ring. Let $A_{0}=A \cap Q(B)$, and put $h=e\left(A A_{0}\right)$. Then we have $r\left(A A_{0}\right) \geq h-1$ by $(\mathrm{i})$ and $r\left(A_{0} B\right) \geq e\left(A_{0} B\right)-1$ by Lemma 7. Thus $r(A B)=$ $r\left(A A_{0}\right)+h r\left(A_{0} B\right) \geq h-1+h\left(e\left(A_{0} B\right)-1\right)=h\left(e\left(A_{0} B\right)\right)-1=e(A B)-1$. (It is easy to see that $e(\bar{A} B)=h\left(e\left(A_{0} B\right)\right)$.) This proves (\#).

We now prove (ii). In the following we assume $Q(A)=Q(B)$. We have $r(A B)=r(Z(A) B)-m+1$ (Lemma 8); thus $r(A B)=n-m$ if and only if $r(Z(A) B)=n-1$, which is equivalent to $Z(A)=Z(B)$ by Lemma 7 .

Finally we assume $Z(A)=Z(B)$. The assertion that $N A=M$ was proved in [S]. We give a simple algebraic proof based upon the inequality $r(A B) \geq$ $e(A B)-\operatorname{dim} A$ obtained above.

Let $E=\left\{b_{1}, \ldots, b_{n}\right\}$ and $E^{\prime}=\left\{a_{1}, \ldots, a_{m}\right\}$ be the minimal bases of $N$ and $M$ respectively. We can choose $a_{i}$ such that $a_{t}, \ldots, a_{m}$ generates $N A \bmod M^{2}$; then $N A=M$ if and only if $t=1$. Let $A^{\prime}$ be the localization of $A\left[a_{2} / a_{1}, \ldots, a_{m} / a_{1}\right]$ at the maximal ideal generated by $E_{0}=$ 
$\left\{a_{1}, a_{2} / a_{1}, \ldots, a_{m} / a_{1}\right\}$. Then $\left(A^{\prime}, A\right)$ is a pair of regular local rings. We calculate $r\left(A^{\prime} B\right)$ and $e\left(A^{\prime} B\right)$.

(a) We have $r\left(A^{\prime} B\right)=r\left(A^{\prime} A\right)+r\left(A^{\prime} A B\right)=m-1+v_{A^{\prime}}(d(A / B))$ where $d(A / B)=(a) A$ with $v_{A}(a)=r(A B)=n-m$. It is easy to see that $v_{A^{\prime}}(g) \leq$ $2 v_{A}(g)$ for any $g \in M$ (using the fact that $E_{0}$ is a minimal basis of the maximal ideal of $\left.A^{\prime}\right)$. Thus $r\left(A^{\prime} B\right) \leq m-1+2(n-m)=2 n-m-1$.

(b) We have $v_{A^{\prime}}\left(a_{i}\right)=2$ for any $1<i \leq m$. Each $b_{i}$ can be written as a linear combination of $a_{t}, \ldots, a_{m} \bmod M^{2}$. Thus if $t>1$ then $v_{A^{\prime}}\left(b_{i}\right) \geq 2$ for all $i$, which implies $e\left(A^{\prime} B\right) \geq 2 n$. Thus $e\left(A^{\prime} B\right)-m \geq 2 n-m>2 n-m-1=$ $r\left(A^{\prime} B\right)$, contradicting the inequality $r\left(A^{\prime} B\right) \geq e\left(A^{\prime} B\right)-m$ of (\#). Thus $t=1$, which means $N A=M$. The proof of the theorem is complete.

\section{REFERENCES}

[A] S. Abhyankar, On the valuations centered in a local domain, Amer. J. Math. 78 (1956), 321-348.

[D] V. I. Danilov, Decomposition of certain birational morphisms, Izv. Akad. Nauk SSSR Ser. Mat. 44 (1980), 465-477; English transl. in Math USSR-Izv. 44 (1980).

[K1] E. Kunz, Die Primidealteiler der Differenten in allgemeinen Ringen, Reine Angew. Math. 204 (1960), 165-182.

[K2] _ Kähler differentials, Adv. Lectures Math., Vieweg, Braunschweig, 1986.

[LL] T. Luo and Z. Luo, Factorization of birational morphisms with fiber dimension bounded by 1, Math. Ann. 282 (1988), 529-534.

[L1] Z. Luo, An invariant approach to the theory of logarithmic Kodaira dimension of algebraic varieties, Bull. Amer. Math. Soc. (N.S.) 19 (1988), 319-323.

[L2] _ Ramification divisor of regular schemes, preprint 1987, Algebraic Geometry Proceeding, Tianjing, Nankai, World Scientific, 1992 (to appear).

[L3] _ Factorization of birational morphisms of regular schemes, Math. Z. 212 (1993), 505-509.

[S] T. Sancho de Salas, Theorems of factorizations for birational morphisms, Compositio Math. 70 (1989), 275-291.

[ZS] O. Zariski and P. Samuel, Commutative algebra, vol. I, Van Nostrand, Princeton, NJ, 1958.

Academia Sinica, Institute of Systems Science, Beijing 100080, People's Republic of CHINA

Current address: 24989 Prospect Avenue, Loma Linda, California 92354 